\title{
Evolution of a multi-agent system in a cyclical environment
}

\author{
Tiago Baptista $\cdot$ Ernesto Costa
}

Received: 11 September 2007/ Accepted: 26 March 2008/Published online: 15 April 2008

(C) Springer-Verlag 2008

\begin{abstract}
The synchronisation phenomena in biological systems is a current and recurring subject of scientific study. This topic, namely that of circadian clocks, served as inspiration to develop an agent-based simulation that serves the main purpose of being a proof-of-concept of the model used in the BitBang framework, that implements a modern autonomous agent model. Despite having been extensively studied, circadian clocks still have much to be investigated. Rather than wanting to learn more about the internals of this biological process, we look to study the emergence of this kind of adaptation to a daily cycle. To that end we implemented a world with a day/night cycle, and analyse the ways the agents adapt to that cycle. The results show the evolution of the agents' ability to gather food. If we look at the total number of agents over the course of an experiment, we can pinpoint the time when reproductive technology emerges. We also show that the agents adapt to the daily cycle. This circadian rhythm can be shown by analysing the variation on the agents metabolic rate, which is affected by the variation of their movement patterns. In the experiments conducted we can observe that the metabolic rate of the agents varies according to the daily cycle.
\end{abstract}

Keywords Artificial life $\cdot$ Emergence $\cdot$ Circadian rhythm

T. Baptista $(\bowtie) \cdot$ E. Costa

CISUC, Department of Informatics Engineering,

University of Coimbra, Polo II, Pinhal de Marrocos,

3030-290 Coimbra, Portugal

e-mail: baptista@dei.uc.pt

E. Costa

e-mail: ernesto@dei.uc.pt

\section{Introduction}

In this paper we describe and analyse the experiments conducted as part of the development of an agent-based simulation model and framework-BitBang-based on principles of complexity science (Menezes et al. 2006). The main drive to build such a system came from noticing that, despite substancial advances in both hardware and software technologies, computer games' artificial intelligence fail to demonstrate the abilities to both surprise the human player for an undetermined period of time and display creative behaviour. Sophisticated as they may be, when played for long enough time, most game AI reveals the deterministic nature of its internal processes. We believe that a system based on complexity science can provide a solution to these shortcomings.

The BitBang framework implements a modern autonomous agent model (Russel and Norvig 1995), with roots in Alife systems. Our world is composed of entities. These can either be inanimate objects which we designate as things, or entities that have reasoning capabilities and power to perceive and affect the world-the agents. Both have traits that characterise them, such as colour, size, or energy-the features. The agents communicate with, and change the environment using perceptions and actions, taking decisions using the brain. In this model, there is no definition of a simulation step, as we wont have any type of centralised control. As such, the simulation is asynchronous. The agents will independently perceive, decide, and act. Moreover, there is no evolutionary mechanism included in the definition of the model. That is because we implement evolution as an action. That is accomplished by giving the agents the capability of reproduction. Again, there is no central control bound to the process of reproduction. The agents choose when to reproduce and with 